\title{
Philosophiques
}

\section{Introduction : Paul Ricoeur (1913-2013)}

Méthode et finitude

\section{Sophie-Jan Arrien}

Volume 41, numéro 2, automne 2014

Centenaire de la naissance de Paul Ricoeur (1913-2013)

URI : https://id.erudit.org/iderudit/1027216ar

DOI : https://doi.org/10.7202/1027216ar

Aller au sommaire du numéro

Éditeur(s)

Société de philosophie du Québec

ISSN

0316-2923 (imprimé)

1492-1391 (numérique)

Découvrir la revue

Citer ce document

Arrien, S.-J. (2014). Introduction : Paul Ricoeur (1913-2013) : méthode et finitude. Philosophiques, 41(2), 233-240. https://doi.org/10.7202/1027216ar d'utilisation que vous pouvez consulter en ligne.

https://apropos.erudit.org/fr/usagers/politique-dutilisation/ 


\title{
INTRODUCTION : Paul Ricœur (1913-2013)
}

Méthode et finitude

\author{
SOPHIE-JAN ARRIEN \\ Université Laval \\ sophie-jan.arrien@fp.ulaval.ca
}

À peine nous avait-il quitté que Ricœur faisait figure de classique du vingtième siècle. L'envergure de sa pensée, avérée au fil des ans, tant par les vastes problèmes qu'il abordait et les traditions philosophiques mobilisées, que par son érudition et son ouverture à moult disciplines non strictement philosophiques laissait toutefois le lecteur, pour ces mêmes raisons, face à une certaine perplexité quant à l'unité et l'originalité propres de sa démarche. C'est au regard de ce double enjeu que j'esquisserai ici, à titre indicatif, une hypothèse de lecture.

Toute démarche philosophique tend à définir les conditions dans lesquelles peut se dérouler la conquête du vrai de telle sorte qu'elle-même respecte ces conditions. De là découle certes sa rigueur, mais aussi son originalité propre. Or, dans la vaste fresque philosophique déployée par Ricœur, c'est en vain semble-t-il qu'on chercherait un discours de la méthode. On nous parlera d'herméneutique, à laquelle Ricœur est immanquablement et à juste titre associé comme l'un de ses meilleurs représentant. Mais en quoi l'herméneutique des symboles de La symbolique du mal, celle du double sens de l'essai sur Freud, celle de la voie longue du Conflit des interprétations, celle que déploie Soi-même comme un autre à titre d'herméneutique du soi, représentent-elles une méthode unitaire à proprement parler? Quel en serait le geste propre, la signature pour ainsi dire? L'œuvre de Ricœur a-t-elle même une signature propre? N'a-t-on pas tous, pour peu qu'on s'y intéresse, entendu fuser à son égard l'accusation d'œcuménisme? Sa pensée ne se dissout-elle pas pour finir, si riche et digne qu'elle soit, au mieux en un dialogue dont on ne saurait tracer les limites, au pire en une forme banale de relativisme? Il nous semble au contraire, serait-ce rétrospectivement, qu'un sens de la méthode, profond et structurant, se dégage bel et bien de l'ensemble.

Toute sa vie, Ricœur est resté à sa façon, sinon phénoménologue stricto sensu, du moins fidèle au principe husserlien voulant que "la vraie méthode [soit] commandée par la nature des objets de la recherche $[\ldots]^{1}{ }^{1}$. En d'autres mots: la méthode doit être déterminée à partir de la chose en question, et non l'inverse. Pour le dire encore dans les termes de Heidegger : «La véritable

1. E. Husserl, Philosophie als strenge Wissenschaft [I9I I], Husserliana XXV, La Haye, Nijhoff, I987, p. 26 (trad. fr. M. de Launay, La philosophie comme science rigoureuse, Paris, PUF, I989). 
méthode surgit [entwächst] [...] du caractère fondamental d'une région d'objets et de sa problématique ${ }^{2}$. La façon dont Ricœur s'est à son tour approprié ce principe me semble pouvoir nous conduire au cœur de son œuvre et de son «style» général.

Quel est en effet «l'objet» de la recherche ricœurienne? Cet objet est essentiellement multiple, voire irrémédiablement éclaté, aurait-on tendance à penser spontanément face à l'abondance des thèmes abordés par Ricœur au fil des années: la volonté, le symbole, le mal, la vérité, l'histoire, l'imagination, la métaphore, la narration, le langage, le temps, le soi, etc., et ce, pour ce qui est de la méthode, en lien tant avec la phénoménologie qu'avec l'histoire de la philosophie, l'herméneutique, le structuralisme, la théorie littéraire, l'historiographie, la psychanalyse, la philosophie analytique, la philosophie politique, etc. Il y a pourtant bien un centre, me semble-t-il, à cette pluralité apparente de champs d'investigation, à savoir "l'homme" qui se découvre «soi ». De ce point de vue, la pensée ricœurienne pourrait s'identifier de façon générale à une anthropologie - elle-même raccordée à une herméneutique du soi. Mais quelle serait plus précisément la «nature » (Husserl) ou le "caractère fondamental» (Heidegger) de l'homme (à titre d'objet de la recherche) duquel devront s'originer tant la problématique que la méthode de l'investigation? Ici encore un mot s'impose: celui de finitude. De La philosophie de la volonté (1950) au Parcours de la reconnaissance (2004), de l'homme faillible à l'homme capable, la finitude de l'homme dans ses capacités à agir et à imaginer, à se comprendre et à se raconter, à connaître et à promettre, à penser le vrai et à réaliser le juste, à se souvenir et à pardonner, est ce que Ricœur a tenté de penser. L'homme fini ou la finitude humaine, tel est le foyer de l'anthropologie de Ricœur sous ses figures diverses.

Si l'on m'accorde ces prémisses, on m'accordera également, conformément au principe phénoménologique énoncé plus haut, que la méthode, s’il en est une chez Ricœur, devra être définie en lien avec la problématique névralgique de la finitude ou, plus exactement, qu'elle devra «surgir» de cette problématique. Or tel est précisément le cas:

C'est vrai que je vais peut-être d'une philosophie affirmative, assez sûre d'ellemême, vers une philosophie beaucoup plus interrogative. Vous pouvez appeler ça relativisme, mais moi je n'aime pas tellement le mot «relativisme». Moi j'introduirais plutôt le mot "finitude». Je sais que j'ai une perspective limitée sur les problèmes et que d'autres ont une autre perspective. J'appartiens à une communauté philosophante où j'admets que d'autres voient des choses que moi je ne vois pas. C'est vrai, vous pouvez appeler ça «relativisme », mais c'est

2. M. Heidegger, Grundprobleme der Phänomenologie [I919/20], Gesamtausgabe T. 58 [WS I9I9/20], p. 4. 
aussi un acte de confiance dans la capacité des autres de percevoir et de comprendre des choses que je ne comprends pas ${ }^{3}$.

Dans cette citation, tirée d'un entretien datant de I993, Ricœur indique le triple geste de reconnaissance qui fonde sa démarche: il reconnaît la prégnance de l'interrogation sur l'affirmation dans sa pensée pourtant tendue vers le vrai; il reconnaît son appartenance à une "communauté philosophante » qu'il ne peut égaler à lui seul; il reconnaît devoir croire en la capacité des autres "de percevoir et de comprendre des choses» qu'il ne comprend pas. Au fondement de ce triple aveu, on trouve pour finir la reconnaissance explicite de la finitude qui est la sienne à titre de philosophe. Finitude qu'il identifie à l'horizon spécifiquement méthodique de sa recherche et qu'il refuse d'assimiler à un simple relativisme.

S'il fallait, maintenant, nommer l'indice concret du "principe de finitude» systématiquement à l'œuvre chez Ricœur, le terme de "médiation» serait sans doute le plus approprié. C'est avant tout par un nécessaire travail de médiation que s'atteste la méthode propre à sa philosophie de la finitude. On ne peut, aux yeux de Ricœur, rendre justice philosophiquement à la vérité des choses, du sens, du passé, d'autrui ou de soi-même, si ce n'est par un travail de médiation. Médiation qui s'incarne dans son œuvre par un travail inlassable sur les textes philosophiques (mais pas seulement philosophiques), par une prise en considération des méthodes propres aux différentes disciplines évoquées plus haut et, plus généralement et plus fondamentalement, par une dialectique de l'appartenance et de la distanciation constamment et diversement reconduite pour rendre compte des chemins qui ramènent le soi à soi ${ }^{4}$. Dans ce travail de médiation, qui est tout aussi bien une traversée des textes que de l'agir humain, pas d'absolue vérité, mais aucun relativisme; bien plutôt, une prise au sérieux de la finitude qui échoit à l'homme à tous les niveaux: ontologique, existentiel, temporel, relationnel, narratif, éthique, historique, langagier, mais aussi épistémologique et gnoséologique.

On notera à cet égard que le travail de la médiation chez Ricœur est souvent décrit comme une pratique du "dialogue» interphilosophique ou interdisciplinaire élevé au rang de "style» philosophique ${ }^{5}$. Sans vouloir le moindrement nier la dimension "dialogique» de la démarche ricœurienne, il s'agira ici de compléter cette dernière par quelques considérations qui

3. P. Ricœur et Yvanka B. Raynova, "Quo vadis? Un entretien avec Paul Ricœur" [1993], Labyrinth, vol. 2, hiver 2000 (http://labyrinth.iaf.ac.at/200o/ricoeur.html).

4. Pour une étude éclairante de la vaste portée de la dialectique entre appartenance et appropriation chez Ricœur, on lira avec profit l'étude de Marc-Antoine Vallée, Gadamer et Ricœur. La conception herméneutique du langage (Rennes, Presses universitaires de Rennes, 20I2), en particulier le troisième chapitre.

5. Voir par exemple l'ouvrage de J. Porée et G. Vincent, Paul Ricœur. La pensée en dialogue, Rennes, Presses universitaires de Rennes, 2010. 
permettront peut-être d'en fonder le caractère proprement méthodique. Car le fait est que le «dialogue herméneutique», pour prégnant et structurant qu'il semble être dans son œuvre, n'a pas été formalisé par Ricœur dans un quelconque "discours de la méthode». Or l'esquisse d'une telle formalisation, fût-elle rétrospective, m'apparaît possible si l'on quitte précisément le champ lexical du dialogue pour mettre en avant celui du témoignage. Cette analogie n'aurait aucun avantage sur celle du dialogue, bien au contraire, si ce n'était du fait que l'on retrouve, avec la question du témoignage, la dimension de la finitude rapportée cette fois-ci à l'horizon de tout travail méthodique en philosophie, à savoir la question de la vérité elle-même, comprise par Ricœur comme attestation.

C'est sans doute dans la préface de Soi-même comme un autre qu'est le mieux défini ce qui caractérise le témoin, et simultanément son témoignage en lien avec la notion d'attestation. Celle-ci apparaît sous la plume de Ricœur comme le "mode aléthique (ou véritatif) du style [...] approprié à l'herméneutique du $\operatorname{soi}^{6}$ ». L'attestation désigne ainsi le type de certitude à laquelle peut prétendre la démarche de Ricœur. Non pas la certitude ultime et auto-fondatrice du cogito cartésien, mais une certitude plus fragile conçue comme «fiance» et «créance» (ou croyance):

L'attestation, en effet, se présente d'abord comme une sorte de croyance. Mais ce n'est pas une croyance doxique, au sens où de la doxa - la croyance - a moins de titre que l'épistémè - la science, ou mieux le savoir. Alors que la croyance doxique s'inscrit dans la grammaire du «je-crois-que », l'attestation relève de celle du «je-crois-en». Par là elle se rapproche du témoignage, comme l'étymologie le rappelle, dans la mesure où c'est en la parole du témoin que l'on croit ${ }^{7}$.

À la lumière de ce rapprochement fait par Ricœur lui-même entre attestation et témoignage, et par-delà la stricte herméneutique phénoménologique déployée dans Soi-même comme un autre (où "l'attestation est fondamentalement attestation $\left.d e \mathrm{soi}^{8} »\right)$, il nous semble possible d'utiliser, de façon certes analogique, les ressources de la notion d'attestation pour aiguiser notre intelligence du geste philosophique plus général qui caractérise la démarche du philosophe.

Il s'agit ici de considérer que Ricœur interpelle les auteurs non pas d'abord et uniquement comme les interlocuteurs d'un dialogue fictif, mais qu'il les appelle à la barre, pour ainsi dire, comme autant de témoins «crédibles » ou "fiables ", à qui il est demandé d'exposer leur «version des faits " et du même coup apporter un éclairage valable (mais non exclusif ni définitif) sur le problème et la question en jeu: le soi, le temps, l'agir, le pâtir,

6. P. Ricœur, Soi-même comme un autre, Paris, Seuil (coll. «Point»), I990, p. 33.

7. Ibid.

8. Ibid., p. 34 . 
l'amitié, la justice, la reconnaissance, etc. Or un témoin n'est jamais choisi de façon arbitraire, mais bien parce qu'il apporte une perspective pertinente, parce que ce dont il atteste est propre à éclairer une situation ou un problème non résolu, à propos desquels d'ailleurs nulle certitude définitive n'est permise. On n'apprend donc quelque chose du témoin et on n'accepte d'en apprendre quelque chose que dans la mesure où l'on croit en sa parole et où on lui accorde préalablement sa (con)fiance: "vous pouvez appeler ça "relativisme", mais c'est aussi un acte de confiance dans la capacité des autres de percevoir et de comprendre des choses que je ne comprends pas ${ }^{9}{ }^{\prime}$. Chaque témoin aura permis d'instruire la cause, mais il n'y aura jamais de garantie que son témoignage soit le fin mot de l'affaire; la possibilité d'un meilleur témoin demeure toujours. Telle est la vulnérabilité spécifique du témoignage en son caractère véritatif, lui-même renvoyé à l'attestation hantée par le soupçon comme par son envers. Par là, affirme Ricœur, «la parenté entre attestation et témoignage se vérifie [...]: il n'y a pas d'autre recours contre le faux témoignage qu'un autre témoignage plus crédible; il n'y a pas d'autre recours contre le soupçon qu'une attestation plus fiable ${ }^{10}$ ».

Ainsi se dégage, à l'aune du témoignage et de la vérité comme attestation, une indication pour un discours de la méthode adapté à la pensée ricœurienne - méthode transie de finitude, à l'image de son objet. Il s'agirait, pour le dire avec les mots du philosophe, de la stratégie "d'un discours conscient de son défaut de fondation ${ }^{11}{ }^{\prime}$. Plus que d'engager un simple dialogue herméneutique avec Kant, Hegel, Aristote, Levinas, Husserl, Augustin, Heidegger, Spinoza, Anscombe, Freud ou Beneviste, Ricœur les appelle à la barre sur la foi de leur œuvre respective. Et, (contre-)interrogeant ses témoins, il avance dans ses propres questions, quitte à se heurter à une aporie et à devoir reprendre ailleurs. Cette posture, Ricœur la revendique, clairement du reste, quand il parle «d'aporie productive ${ }^{12}$ » ou admet être passé à « une philosophie [...] interrogative » où l'accent est mis «sur les apories et l'aporétique $»^{13}$.

À ce rythme toutefois, ce sont vite les questions elles-mêmes et pas seulement les réponses qui évoluent. Or telle est bien la marque du travail ricœurien porté par cette méthode qui, de questions en problèmes, de problèmes en apories et d'apories en questions, ne cherche pas tant à refermer ces dernières qu'à les développer et les approfondir: "Je crois qu'avant de

9. P. Ricœur et Yvanka B. Raynova, «Quo vadis? Un entretien avec Paul Ricœur» [I993], Labyrinth, vol. 2, hiver 2000, je souligne (http://labyrinth.iaf.ac.at/2000/ricoeur.html).

10. P. Ricœur, Soi-même comme un autre, Paris, Seuil, I990, p. 34.

11. Ibid.

12. Voir, notamment, Lectures 3: Aux frontières de la philosophie, Paris, Seuil, 2006, p. $23 \mathrm{I}$.

13. P. Ricœur et Yvanka B. Raynova, "Quo vadis? Un entretien avec Paul Ricœur» [1993], Labyrinth, vol. 2, hiver 2000 (http://labyrinth.iaf.ac.at/2000/ricoeur.html). 
parler de l'évolution des réponses il faut parler de l'évolution des questions ${ }^{14}$.»

C'est donc précisément sous le sceau de "l'évolution des questions" que j'ai choisi de placer le présent dossier. N'y sont pas tant reprises les confrontations interphilosophiques et interdisciplinaires que Ricœur a constamment entretenues avec les uns et les autres, que l'évolution des questions que ces confrontations ont rendu possible. Les six textes qui composent le dossier se concentrent, comme autant de questions ouvertes, sur quelquesuns des grands axes thématiques de l'anthropologie philosophique de Ricœur: l'imagination, l'agir, l'expérience du temps, le soi et l'autre, les thèmes des passions et de l'aveu. Chacun des textes déploie, à sa propre manière, l'évolution des questions à partir de laquelle la réflexion ricœurienne s'est, jusqu'à son terme, enrichie en nuances, en complexité et en profondeur.

Dans un texte consacré à l'imagination, Rodolphe Calin relève une certaine hésitation, qui, par moments, devient tension dans l'œuvre de Ricœur quant à l'articulation des règnes de l'image et du langage qui s'y fait jour. En effet, si un certain primat du langage sur l'image traverse la sémantique de l'image mise en œuvre par De l'interprétation (1965) et La métaphore vive (I975), les choses semblent s'infléchir dans l'autre direction dans le texte "Image et langage dans la psychanalyse » (I978) qui met plutôt l'accent sur une sémiotique de l'image. C'est au cœur de cette tension qu'il rend dès lors productive que s'installe l'auteur pour déployer le concept ricœurien d'imagination créatrice.

Reprenant à son tour la question de l'imagination chez Ricœur dans un texte consacré au rôle pratique de celle-ci, Michaël Foessel dégage le lien fondamental que le philosophe a très tôt identifié entre l'imaginaire et l'action. Du projet d'une "poétique de la volonté » au déploiement d'une herméneutique des capacités, l'imagination se voit comprise de plus en plus précisément comme la faculté de se rapporter au réel (tant à travers le récit qu'à travers la métaphore) sous le mode de la contre-factualité, et dès lors comme la faculté d'affecter ce dernier de l'indice du projet. Cette prise sémantique sur le réel qui n'est ni de l'ordre de la perception ni de l'ordre du concept, mais qui articule ces deux dimensions, devient, comme le fait valoir l'auteur, un pouvoir pratique, un pouvoir d'innover et d'ouvrir pour le monde un horizon «utopique » où le sujet projette ses possibilités propres.

Le texte de Marie-Hélène Desmeules reprend et approfondit, compte tenu des "glissements" qu'elle y découvre, le thème que j'ai nommé "prise sémantique sur le réel » eu égard à l'expérience fondamentale du temps. De Temps et récit à La mémoire, l'histoire, l'oubli, elle montre l'évolution des différentes médiations objectives qui, par-delà la dimension narrative, en viennent à participer, sous des modes non seulement positifs mais aussi

14. P. Ricœur et Yvanka B. Raynova, "Quo vadis? Un entretien avec Paul Ricœur» [1993], Labyrinth, vol. 2, hiver 2000 (http://labyrinth.iaf.ac.at/200o/ricoeur.html). 
négatifs, à la transformation du sujet et de ses possibilités, y compris et de façon névralgique, au lieu le plus intime de sa configuration temporelle.

Portant lui aussi un regard rétrospectif sur l'œuvre de Ricœur mais dans une perspective qui tient davantage de la reconstruction, Jean-Sébastien Hardy part du projet d'une phénoménologie des passions, esquissée puis abandonnée par le jeune Ricœur, pour montrer que le philosophe a malgré tout, dans son œuvre ultérieure, repris la question. Ricœur s'avère ainsi avoir non seulement dégagé les ressources conceptuelles nécessaires pour prendre en considération l'affectivité passionnelle en son intentionnalité spécifique, mais tenté de penser le rôle constituant de certaines passions fondamentales dans notre rapport au monde vécu.

On retrouve de nouveau les réflexions du jeune Ricœur au point de départ du texte de Simon Castonguay. Celui-ci y analyse, à titre de porte d'entrée de l'herméneutique ricœurienne, le thème de l'aveu développé dans la Symbolique du mal (I960). Plutôt toutefois que de retracer le destin de l'aveu à même le corpus de Ricœur, il choisit de faire évoluer la question en la confrontant à la pensée de Michel Foucault pour qui l'aveu, source également d'une certaine herméneutique du soi, doit cependant être pensé comme technique de la gouvernementalité. Dès lors, l'auteur tente de rendre manifeste le spectre des enjeux liés à la question de l'aveu selon qu'elle est l'index soit d'une anthropologie de la finitude, soit d'une généalogie du pouvoir.

Finalement, c'est en confrontant lui aussi Ricœur à ses contemporains que Marc-Antoine Vallée ressaisit la question du soi, question transversale s'il en est une chez le philosophe. Il identifie ainsi chez Levinas et Marion une tendance, qu'il fait remonter à Kierkegaard et qui consiste à dissoudre tout danger d'absolutisation du sujet dans la promotion d'une « intelligence relationnelle» du soi. Seulement, ces approches courent à leur tour le danger, sinon de dissoudre le soi, du moins de le priver de toute consistance propre. À l'encontre de cette tendance, l'auteur identifie avec et par-delà Ricœur les dimensions déterminantes sinon constituantes du soi que sont les relations, les appartenances et les capacités.

$$
* * *
$$

En 2013 fut commémoré le centième anniversaire de naissance de Paul Ricœur. Les manifestations rendant hommage au philosophe furent nombreuses. Les colloques internationaux se succédèrent, et la pensée de Ricœur s'y trouva discutée comme jamais, témoignant de l'intérêt qu'elle ne cesse de susciter dans la communauté philosophique. C'est dans ce contexte qu'a eu lieu, à l'Université Laval, le 29 mars 20I3, une journée d'étude consacrée à la philosophie de Paul Ricœur. Le choix de tenir cette rencontre au Québec n'est pas insignifiant au regard des liens privilégiés qui unissaient Paul Ricœur à la Belle Province. En effet, comme l'a alors rappelé Jean Grondin en conférence d'ouverture, Ricœur fut, dès le tout début des années soixantedix et jusqu'au seuil de l'an deux mille, plusieurs fois sollicité comme professeur invité ou comme conférencier à l'Université de Montréal, où un 
poste de professeur lui fut même proposé, qu'il déclina finalement ${ }^{15}$. C'est donc l'ami du Québec et l'immense philosophe que cette journée et le présent dossier ont voulu honorer ${ }^{16}$.

15. Voir le texte complet de l'intervention de Jean Grondin: http://www.fabula.org/ colloques/documentr9i 6.php

16. Les contributions qui furent présentées lors de cette journée ont inspiré la majorité des travaux présentés dans ce dossier. 